Session 4: Theory of stellar evolution \& atmospheres: beyond standard physics, rotation, duplicity, mass loss and magnetic fields and instabilities 


\title{
Evolution models of red supergiants
}

\author{
Cyril Georgy \\ Department of Astronomy, University of Geneva, \\ Chemin des Maillettes 51, 1290 Versoix, Switzerland \\ email: cyril.georgy@unige.ch
}

\begin{abstract}
The red supergiant (RSG) phase is a key stage for the evolution of massive stars. The current uncertainties about the mass-loss rates of these objects make their evolution far to be fully understood. In this paper, we discuss some of the physical processes that determine the duration of the RSG phase. We also show how the mass loss affect their evolution, and can allow for some RSGs to evolve towards the blue side of the Hertzsprung-Russell diagram. We also propose observational tests that can help in better understanding the evolution of these stars.
\end{abstract}

Keywords. stars: evolution, stars: interiors, stars: luminosity function, stars: mass loss, supergiants, convection

\section{Introduction}

The red supergiant (RSG) phase is an important phase of the life of massive stars. Indeed, a significant fraction of all massive stars will spend some time in this stage. Moreover, some of them will finish their life as a RSG, and explode as a type II-P supernova (SN), which represents about half of the total number of core-collapse SNe (Smith et al. 2011). The RSG phase occurs because at the end of the main sequence (MS), the core contraction will lead to a huge extension of the envelope (the so-called "mirror effect", see e.g. Kippenhahn \& Weigert 1990). This makes the star evolve redwards in the Hertzsprung-Russell diagram (HRD), up to the point where the effective temperature of the star is $\log \left(T_{\text {eff }}\right) \sim 3.5-3.6$. However, the modelling of the crossing of the HRD is strongly dependent on the way several physical processes are implemented into stellar evolution codes (e.g. convection, or rotation-induced instabilities).

During the RSG phase, the major uncertainty from the point of view of stellar evolution modelling is the mass loss. Mass loss from RSGs is still not understood on a theoretical point of view, so there is so far no equivalent to the radiative wind theory (e.g. Castor et al. 1975), established for hot stars, for this cool phase. The mass-loss rates of RSGs rely thus on observational determinations, which can vary over orders of magnitude at a given luminosity or effective temperature (see below).

The choice of weak or strong mass-loss rates during the RSG phase affects dramatically the modelling of the advanced stages of a massive star. In some cases, it is possible to make the star leaves the RSG branch and starts a new crossing of the HRD, becoming again a blue supergiant (BSG). In this paper, we illustrate some of these difficulties in modelling RSG, and how it is affected by the treatment of convection and mass-loss in stellar evolution codes. In section 2, we discuss briefly the first crossing of the HRD at the end of the MS. In section 3, we show the impact of the choice of the mass-loss rates during the RSG phase on the subsequent evolution. Finally, we discuss a few observational tests that could be used to test stellar evolution codes in section 4 . 


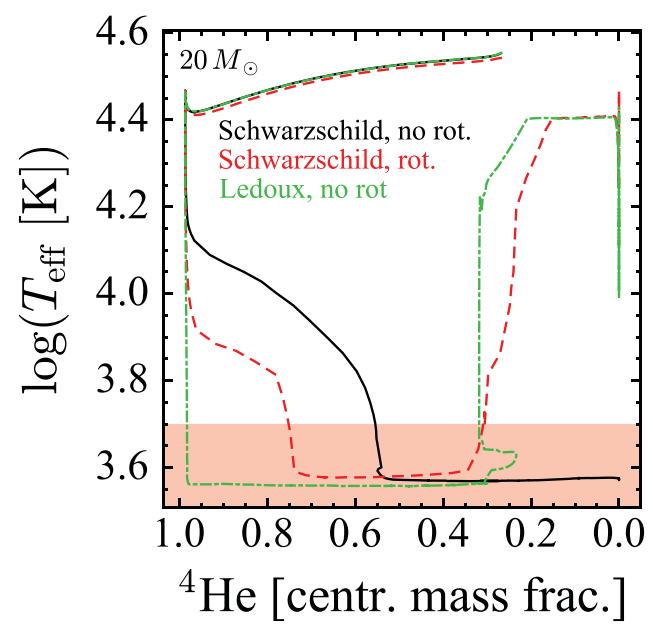

Figure 1. Tracks of $20 M_{\odot}$ stars at solar metallicity in the $\log \left(T_{\text {eff }}\right)$ vs. central helium mass fraction plane, with different inputs physics. The solid line is a non-rotating model computed with the Schwarzschild criterion for convection. The long-dashed one includes the effect of rotation (initial velocity corresponding to 0.4 the critical one). The dot-dashed line is a non-rotating model computed with the Ledoux criterion for convection. The ZAMS is located on the top. The models then proceed along the MS towards the left, up to the point where the central helium mass fraction reaches almost 1 . The tracks then cross the HRD, entering the red zone of the plot, illustrating the RSG region. At the end of their life, the rotating and Ledoux model cross again the HRD, reaching higher effective temperature, while the non-rotating Schwarzschild model remains in the RSG region.

\section{First crossing of the HRD}

Figure 1 illustrates the sensitivity of the first crossing of the HRD on the implemented physics inside a stellar evolution code in the $\log \left(T_{\text {eff }}\right)$ vs. central helium mass fraction plane. The figure shows the tracks of three different models of solar metallicity $20 M_{\odot}$ stars: a non-rotating model computed with the Schwarzschild criterion for convection (solid line), a rotating model with the same criterion for convection (long-dashed line), and a non-rotating model computed with the Ledoux criterion for convection (dot-dashed line). For all three models, the ZAMS is located on the top, where all three curves are merged. The evolution proceeds towards the left, the central He mass fraction increasing to 1 during the MS. Then the tracks cross the HRD with different behaviour, reaching the region highlighted in red, corresponding to the effective temperatures of RSGs. The crossing of the HRD occurs very differently according to the physical assumptions that are made: the non-rotating Schwarzschild model crosses slowly, becoming a RSG only when it has burned about $45 \%$ of its central helium content. On the other hand, the model computed with the Ledoux criterion crosses directly the HRD before starting burning helium as a RSG.

These different behaviours are linked to the position and activity of an intermediate convective zone appearing on top of the former hydrogen-burning core at the end of the MS. The way both rotation and convection are implemented influences this intermediate convective shell. When it is active enough, it can sustain the luminosity of the star for a while, making the crossing occurs slowly. When it is less active, the star contracts more quickly, producing a fast crossing of the HRD.

These behaviours have important consequences on various aspects of the post-main sequence evolution of the star. It changes the time spent in the blue or red side of the HRD. It will thus affect the blue- to red-supergiant ratio in stellar population, ratio 


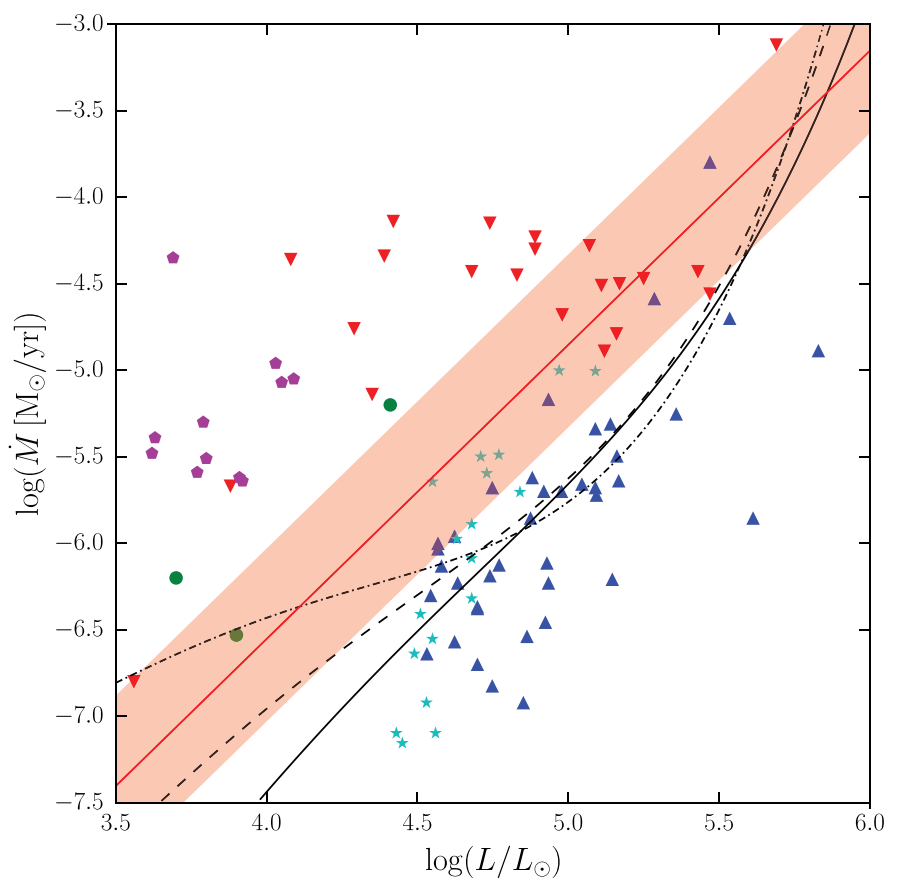

Figure 2. Mass-loss rates of RSGs deduced by different authors: Mauron \& Josselin (2011, dark blue up-triangles), M-type (red down-triangles), MS-type (green circles), and carbon RSGs (purple pentagons) from van Loon et al. (2005) and RSGs from Beasor \& Davies (2016, cyan stars). The three curves are mass-loss rates computed with the de Jager et al. (1988) recipe, for effective temperature of $\log \left(T_{\text {eff }}\right)=3.5$ (solid line), 3.6 (dashed line) and 3.7 (dot-dashed line). The shaded area shows the mass-loss rate used in the Geneva stellar evolution code (from observations from Crowther 2001): standard rate follows the bottom boundary of the shaded area, the middle line represents a mass-loss rate multiplied by a factor of 3 , and the top boundary by a factor of 10 .

which is still poorly reproduced by stellar models as of today (Langer \& Maeder 1995; Eggenberger et al. 2002, see also Eldridge et al. 2008). By changing the duration of the RSG phase, it modifies the total amount of mass lost during this stage. Also, the way the HRD is crossed affects the time at which a possible Roche-lobe overflow can occur in case of a binary system.

\section{Impact of the mass-loss rates on the advanced stages of massive star life}

Figure 2 shows the mass-loss rates deduced observationally from different samples of RSG (Mauron \& Josselin 2011; van Loon et al. 2005; Beasor \& Davies 2016). From this plot, we see that the classical mass-loss recipes used in stellar evolution codes (often from de Jager et al. 1988) fit well the bulk of RSGs with the lowest mass-loss rates. However, some stars exhibit a much higher rate (more than 1 order of magnitude). It is not clear yet why so high mass-loss rates are present: is it linked to a peculiar evolutionary phase of the star? Is the duration of this high mass-loss rate phase long enough to have an impact on the total mass lost during the RSG phase? How to include it in stellar evolution codes?

This has motivated various studies, showing how an increased mass-loss rate during the RSG phase affects the subsequent evolution of the star. First of all, an increased mass-loss rate allows for some massive stars (typically with an initial mass 


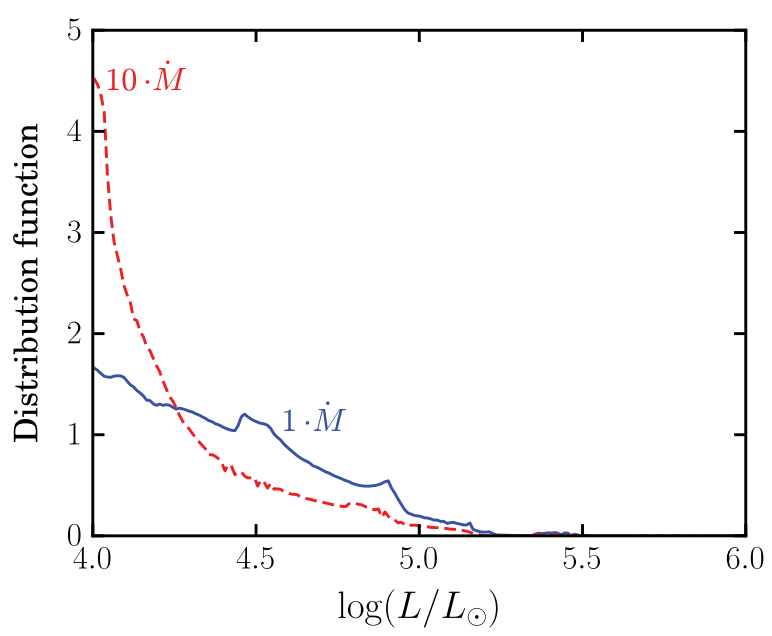

Figure 3. Luminosity distribution function for single RSG for two different mass-loss rates: computed with models using the standard mass-loss rate (blue solid line) and using a mass-loss rate increased by a factor of 10 during the RSG phase (red dashed line). From Georgy \& Ekström (2016).

higher than about $\left.20 M_{\odot}\right)$ to cross the HRD for a second time after the RSG phase (Vanbeveren et al. 1998a,b). This is a possible solution for the so-called "red supergiant problem" (Smartt et al. 2009, see also Georgy et al. 2012; Walmswell \& Eldridge 2012): no type II-P supernova (SN) progenitor is found with an initial mass higher than about $17 M_{\odot}$, while higher mass RSGs are known, which should explode as a type II-P SN. A solution to this problem is thus that higher initial mass RSGs evolve towards the blue side of the HRD before exploding.

In Georgy (2012), we have discussed the impact of an increased mass-loss rate during the RSG phase for stars with an initial mass of about $12-15 M_{\odot}$, and shown that their end-point in the HRD is located inside the yellow supergiant region. Their very small hydrogen-rich envelope would make it appears as a type IIb or II-L SN at the time of the explosion. In case high $\dot{M}$ are possible for RSGs in this mass range, this could be an alternative scenario to the classical binary channel scenario (e.g. Podsiadlowski et al. 1993; Claeys et al. 2011; Benvenuto et al. 2013) for producing yellow supergiant SN progenitors.

Meynet et al. (2015a) have studied in more details the impact a higher $\dot{M}$. They have shown that using a large mass-loss rate indeed decreases the time spent as a RSG. Interestingly, however, it appears that the total amount of mass lost during the RSG phase is almost not affected by the choice of the mass-loss rate for RSG stars evolving back to blueside of the HRD.

The change in the duration of the RSG phase at different mass for different mass-loss rates has, as a direct consequence, a modification of the luminosity distribution function of such stars. This is illustrated in Fig. 3. Indeed, as discussed in Meynet et al. (2015a), a high mass-loss rate tends to remove the Cepheid loops in the intermediate mass star regime, making them spending more time in the RSG phase. On the other hand, a higher mass-loss rate will decrease the duration of the RSG phase for stars with a mass of about $20-25 M_{\odot}$, by making them evolving back to the blue side of the HRD. This makes the luminosity distribution more peaked at lower luminosity for higher mass-loss rates during the RSG phase. 


\section{A second population of BSGs?}

As mentioned in the previous section, a high mass-loss rate during the RSG phase favours a blueward evolution. This occurs when the mass of the core becomes an important fraction of the total mass of the star (typically more than about $60 \%$, see Giannone 1967). In case such kind of evolutionary path would exist, we can wonder whether there is a way to distinguish those BSGs coming back from the red side of the HRD after the RSG phase (hereafter group 2 BSGs) and the classical BSGs that are stars on their first crossing of the HRD (hereafter group 1 BSGs).

In Saio et al. (2013), we have shown that group 1 and group 2 BSGs have very different pulsational properties. Indeed, due to the strong mass loss during the RSG phase, group 2 stars have a much higher luminosity to mass ratio, favouring excitation of pulsations inside these stars. The period of radial pulsations in our models of group 2 BSGs are qualitatively in good agreement with the observed period of variability of $\alpha$ Cygni variables, indicating that this class of variable stars could be post-RSG stars. However, these models, computed with the Schwarzschild criterion for convection, have a surface chemical composition strongly processed by CNO cycle, with for example N/C ratio up to 60 . This is not compatible with the observed value of about 2-3 for the $\alpha$ Cygni variable stars Rigel and Deneb (Przybilla et al. 2010).

In a following study (Georgy et al. 2014), we have shown that using the Ledoux criterion instead of the Schwarzschild one improves a lot the situation. Indeed, the position of the intermediate hydrogen-burning convective zone is shifted at a higher position inside the star with the Ledoux criterion. This prevents the transport strongly CNO-processed material close enough to the surface. However, we still have some difficulties in reproducing the observed surface gravity for these stars (see also Saio et al. 2015).

Another interesting property of BSGs is that they lie in a tight linear relation in the bolometric magnitude vs $\log \left(g / T_{\text {eff }}^{4}\right)$ plane (called the "flux-weighted gravity - luminosity relation", FWLR, see Kudritzki et al. 2003). In Meynet et al. (2015b), we have studied how population of stars containing both group 1 and group 2 BSGs fits this relation. It appeared that the FWLR is not compatible with a lot of objects of the group 2 . However, the general agreement between our models and the observed FWLR is quite good. The next step in this direction will be to include the effects of binary evolution in this diagram.

\section{Conclusions}

In this short paper, we have discussed the way a massive star crosses the HRD at the end of the MS to become a RSG, and show the sensitivity of this crossing on the way convection or rotation is implemented inside stellar evolution code. Once the star is a RSG, its mass-loss rate is a key ingredient in determining what will be its future evolution. In the most extreme cases, the star loses enough mass to cross the HRD a second time, becoming again a BSG. We propose different ways to observationally constrain the physics (particularly, the physics of convection and the average mass-loss rate during the RSG phase) in the models: the luminosity distribution of RSG, the pulsational properties of BSGs and the FWLR can provide useful informations about the RSG stage.

\section{References}

Beasor, E. R. \& Davies, B. 2016, MNRAS 463, 1269

Benvenuto, O. G., Bersten, M. C., \& Nomoto, K. 2013, ApJ 762, 74

Castor, J. I., Abbott, D. C., \& Klein, R. I. 1975, ApJ 195, 157 
Claeys, J. S. W., de Mink, S. E., Pols, O. R., Eldridge, J. J., \& Baes, M. 2011, A\&A 528, A131

Crowther, P. A. 2001, in D. Vanbeveren (ed.), The Influence of Binaries on Stellar Population Studies, Vol. 264 of Astrophysics and Space Science Library, p. 215, Kluwer Academic Publishers, Dordrecht

de Jager, C., Nieuwenhuijzen, H., \& van der Hucht, K. A. 1988, A\& $A S$ 72, 259

Eggenberger, P., Meynet, G., \& Maeder, A. 2002, A\&A 386, 576

Eldridge, J. J., Izzard, R. G., \& Tout, C. A. 2008, MNRAS 384, 1109

Georgy, C. 2012, A\&̈A 538, L8

Georgy, C. \& Ekström, S. 2016, IAU Focus Meeting 29, 454

Georgy, C., Ekström, S., Meynet, G., et al. 2012, A\&A 542, A29

Georgy, C., Saio, H., \& Meynet, G. 2014, MNRAS 439, L6

Giannone, P. 1967, ZAp 65, 226

Kippenhahn, R. \& Weigert, A. 1990, Stellar Structure and Evolution, Stellar Structure and Evolution, XVI, 468 pp. 192 figs. Springer-Verlag Berlin Heidelberg New York. Also Astronomy and Astrophysics Library

Kudritzki, R. P., Bresolin, F., \& Przybilla, N. 2003, ApJ 582, L83

Langer, N. \& Maeder, A. 1995, A\&A 295, 685

Mauron, N. \& Josselin, E. 2011, A\&A 526, A156

Meynet, G., Chomienne, V., Ekström, S., et al. 2015a, A\&A 575, A60

Meynet, G., Kudritzki, R.-P., \& Georgy, C. 2015b, A\&A 581, A36

Podsiadlowski, P., Hsu, J. J. L., Joss, P. C., \& Ross, R. R. 1993, Nature 364, 509

Przybilla, N., Firnstein, M., Nieva, M. F., Meynet, G., \& Maeder, A. 2010, A\& 4 517, A38+

Saio, H., Georgy, C., \& Meynet, G. 2013, MNRAS 433, 1246

Saio, H., Georgy, C., \& Meynet, G. 2015, in G. Meynet, C. Georgy, J. H. Groh, \& Ph. Stee (ed.), IAU Symposium, Vol. 307 of IAU Symposium, pp 230-231

Smartt, S. J., Eldridge, J. J., Crockett, R. M., \& Maund, J. R. 2009, MNRAS 395, 1409

Smith, N., Li, W., Filippenko, A. V., \& Chornock, R. 2011, MNRAS 412, 1522

van Loon, J. T., Cioni, M.-R. L., Zijlstra, A. A., \& Loup, C. 2005, A\&A 438, 273

Vanbeveren, D., De Donder, E., van Bever, J., van Rensbergen, W., \& De Loore, C. 1998a, New A 3,443

Vanbeveren, D., De Loore, C., \& Van Rensbergen, W. 1998b, A\&A Rev. 9, 63

Walmswell, J. J. \& Eldridge, J. J. 2012, MNRAS 419, 2054 